Voix et Images

\title{
L'écriture du désir. Entretien avec Madeleine Ouellette-Michalska
}

\section{Janet M. Paterson}

Volume 23, numéro 1 (67), automne 1997

Madeleine Ouellette-Michalska

URI : https://id.erudit.org/iderudit/201340ar

DOI : https://doi.org/10.7202/201340ar

Aller au sommaire du numéro

Éditeur(s)

Université du Québec à Montréal

ISSN

0318-9201 (imprimé)

1705-933X (numérique)

Découvrir la revue

Citer ce document

Paterson, J. M. (1997). L'écriture du désir. Entretien avec Madeleine

Ouellette-Michalska. Voix et Images, 23(1), 11-24.

https://doi.org/10.7202/201340ar d'utilisation que vous pouvez consulter en ligne.

https://apropos.erudit.org/fr/usagers/politique-dutilisation/ 


\title{
L'écriture du désir. Entretien avec Madeleine Ouellette-Michalska
}

\author{
Janet M. Paterson, Université de Toronto
}

V. et I.: Quels éléments de votre enfance et de votre adolescence vous ont menée à l'écriture et ont marqué votre œuvre?

M. O.-M. : Il y a eu beaucoup de choses et, à la fois, peu de choses. Dans le beaucoup de choses, le contact avec les grands espaces vibrants de lumière - je parle de l'été naturellement, car je détestais l'hiver - m'a donné une sorte de spatialisation du monde, du temps, de la pensée. À la campagne, le temps est beaucoup plus marqué, les saisons sont très détachées; l'hiver, les fenêtres du premier étage étaient couvertes de neige. Cet espace créait un effet d'isolement. J'ai souvent éprouvé une sorte de réclusion, car les voisins étaient loin. Comme je n'ai pas eu la socialisation par le jeu et par l'école, je suis restée une grande sauvage solitaire, même si j'ai toujours exercé des métiers de communication. La nature m'a donné le sentiment du rapport entre toutes choses. Je gravissais souvent ce qu'on appelait le cap à côté de la maison, car on habitait sur la route de Gaspé, et là j'avais l'impression de voir le monde. Le fleuve n'était pas là, mais je l'ai trouvé à l'adolescence quand on a déménagé à Rivière-duLoup, une petite ville de 18000 habitants. C'était la première fois que, de façon tangible, je goûtais le sentiment de la liberté. Une splendeur.

V. et I. : Est-ce vrai que vous avez eu une enfance non scolarisée?

M. O.-M. : Presque. J'ai fréquenté le couvent du village un an et c'était un grand bonheur. Je suis née à la campagne, dans une famille de quinze enfants, de parents scolarisés qui aimaient lire. Mais pour différentes raisons, dont des difficultés socio-économiques - car il y a eu déclin d'année en année de la propriété achetée par mon grand-père paternel -, je n'ai pas fréquenté l'école. Mais depuis que j'ai de la mémoire, j'ai eu deux aspirations. L'aspiration à la liberté et l'aspiration d'apprendre. J'ai donc appris: on n'apprend pas qu'à l'école. J'ai commencé à enseigner aux enfants des voisins, ce dont mon père était très fier, et j'ai ensuite enseigné dans une classe de campagne à 17 ans.

V. et I. : Si vous n'avez pas fréquenté l'école, comment avez-vous appris à lire et à écrire? Est-ce que vous lisiez beaucoup à l'âge de 7 ans, 8 ans? 
M. O.-M.: J'ai appris à lire et à écrire avec mes sœurs, tout particulièrement celle qui me précédait. Par ailleurs, il y avait beaucoup de livres dans la grande maison de trois étages que nous habitions. Le grenier et les armoires étaient remplis de livres qui étaient inappropriés pour moi, des livres d'histoire et des classiques puisque mes tantes avaient fait des études. Il y avait La Rochefoucauld, Racine, Molière et beaucoup d'autres. Je n'y comprenais rien, mais j'étais fascinée par les mots alignés sur la page; fascinée par les tranches dorées, les pages couvertures en couleur ou en cuir. J'éprouvais une véritable passion pour toute cette matière textuelle que je ne comprenais pas d'abord, puis que je suis parvenue à lire.

V. et I. : D'où vient votre intérêt pour l'histoire?

M. O.-M. : J'ai vécu l'expérience contradictoire d'ouverture et de fermeture dès l'enfance et l'adolescence. Le contact avec l'histoire, je l'ai eu très vite puisque les vieilles armoires étaient remplies de livres contenant des récits de bataille dont je lisais des petits bouts. En même temps, l'histoire, je l'entendais par la bouche de ma mère qui connaissait par cœur l'histoire de France et d'Angleterre, les généalogies des grandes familles royales et l'histoire sainte. De son côté, mon père s'intéressait à la politique, alors j'entendais souvent circuler le nom de grands personnages. Mon père avait la passion des idées, de la politique, ma mère avait la passion de la culture et de l'histoire.

V. et I.: Certains de vos essais ainsi que vos textes de fiction sont marqués par ce que vous appelez l'inscription du féminin dans l'écriture. Pouvez-vous tracer votre éveil à cette perspective capitale et son développement?

M. O.-M. : Ce n'est pas venu dans l'enfance, ni même dans l'adolescence. Ma grande préoccupation à cette époque-là, c'était vivre et survivre puis essayer de sauver mes rêves. Certains jours, j'en désespérais totalement. L'aspiration à la liberté était moins urgente pour moi que l'aspiration à la connaissance. Comme ma mère lisait, tenait son journal, j'étais en contact avec une certaine vie intellectuelle, mais je rêvais d'apprendre le savoir des livres. La question de la femme, je ne l'avais pas vraiment en tête. Moi, ce que je voyais, c'étaient des femmes qui se mariaient et qui mettaient des enfants au monde. Je les préférais célibataires. Le jour où elles se mariaient, je me disais: "Se marier, mon dieu, elles vont commencer à faire la cuisine, le ménage, un bébé par année, c'est fini pour elles la vie." V. et I.: L'éveil de votre conscience féminine s'est manifesté à quelle époque alors?

M. O.-M. : Beaucoup plus tard, après mon propre mariage. J'ai commencé ma vie matrimoniale remplie d'une énorme curiosité, mais aussi de tous les stéréotypes acquis sur les rôles masculins et féminins. Auparavant, tout m'avait paru aller de soi en dehors de l'injustice sociale, de la pauvreté et de l'humiliation de ne pas aller à l'école, de ne pas être comme les autres. 
La conscience de la petite fille était là depuis la mémoire, mais la conscience de la femme est venue du savoir et de l'expérience. J'habitais la ville, j'exerçais un métier et j'étudiais. J'étudiais beaucoup, on met du temps à faire les petits points d'une éducation. La conscience de la condition de la femme m'est également venue par le journalisme. Je voyais des femmes de 50 ans, même des femmes de classe bourgeoise, qui tout à coup étaient jetées à la rue, sans protection, sans argent ou qui étaient placées dans des situations de violence aberrante. J'ai voulu trouver la raison de leur aliénation, de leur silence.

V. et I.: Votre vie adulte s'est caractérisée par des expériences qui ont influencé votre vision du monde et, par là même, votre écriture. Dans cette perspective, quel a été l'apport des années que vous avez passées en Algérie?

M. O.-M.: Une perception assez claire de l'existence, de ce que Jung appelait l'inconscient collectif. Dans les ateliers de création que je faisais là-bas, un thème récurrent, avec toutes les futures institutrices auxquelles j'enseignais, était le thème de la marâtre. Quel que soit le sujet suggéré, quelles que soient les pistes où je voulais les envoyer, on arrivait toujours au rituel du mariage, à l'absence d'amour, rituel où la marâtre était constamment présente.

\section{V. et I. : Pourquoi la marâtre?}

M. O.-M. : Parce que les mariages étaient arrangés, l'autorité et l'influence de la marâtre étaient très grandes. Évidemment, dans ce genre de situation, les valeurs affectives étaient difficiles à pratiquer: une civilisation trop contraignante impose des réalités sociales et des schèmes mentaux très durs, car il y a toujours le poids du social sur le privé et sur l'intime. En Algérie, j'ai aussi ressenti l'influence du climat. Il me semble que mon écriture a été plus heureuse là-bas. La Méditerranée africaine est superbe et j'ai été en contact avec des ruines romaines extraordinaires. C'est là que j'ai écrit La femme de sable.

V. et I.: Vous avez mené, quand vous êtes revenue à Montréal, une carrière journalistique très active en présentant, pendant plusieurs années, des chroniques pour Le Devoir et Châtelaine, entre autres, ainsi qu'à Radio-Canada. En quoi ce travail a-t-il influencé votre œuvre?

M. O.-M.: C'est étrange, je dirais que cela m'a beaucoup moins marquée que les séjours à l'étranger et mes études universitaires. C'est plutôt l'inverse qui s'est produit: l'œuvre et les études ont influencé le journalisme culturel que j'ai pratiqué. Cette expérience m'a aidée à écrire plus vite, elle m'a mise en contact avec des gens, des livres que je n'aurais pas connus autrement. Mais pour moi, c'était un prolongement du travail littéraire. Par exemple, j'ai fait beaucoup de documents sur des écrivains pour Radio-Canada. Ce travail s'insérait dans ma passion du livre, ma passion 
des écrivains et de la matière littéraire. En critique littéraire, j'ai tenté de suggérer une autre façon de lire le monde et de lire - au sens médiatique - un livre. Il n'y avait pas de raison que la violence aille de soi, par exemple. Ces chroniques me permettaient de désamorcer des stéréotypes. C'est un travail que j'ai beaucoup aimé et c'est là, je crois, qu'il y a eu le plus d'échos chez les autres.

V. et I. : On a déjà un peu parlé de l'importance de vos études universitaires. D'autodidacte, vous êtes devenue une femme très instruite du point de vue institutionnel, puisque vous détenez une maîtrise et un doctorat en études littéraires. Quel a été l'impact de ces études sur votre profession littéraire?

M. O.-M. : Sur ma profession littéraire... je crois qu'alors je n'avais pas publié de livre, sauf le premier recueil de nouvelles. Je l'avais écrit avant l'université ou pendant les premières années. Je n'étais pas prête, car je voulais d'abord acquérir la liberté et la liberté passait par les études même si la motivation était avant tout intellectuelle. Il y a eu deux phases au cours de mes études.

D'abord, au niveau du baccalauréat à l'université, ce fut un éblouissement total d'être dans une salle de cours avec un vrai prof qui possédait le savoir. C'était un éblouissement enfantin que je garde toujours d'ailleurs face à la connaissance. Et au fur et à mesure que j'avançais, surtout à la maîtrise et au doctorat, le doute a commencé à me saisir. La connaissance m'était venue davantage par des expériences de la vie que par le savoir livresque. Or, ce qu'enseignaient la philosophie, la psychanalyse, l'histoire littéraire, la linguistique, ne me semblait pas toujours cohérent par rapport au réel. Des contradictions flagrantes me sautaient aux yeux, amenant les questions : jusqu'où ces grandes théories sont-elles justes? Comment sontelles pertinentes? De telles questions sont devenues rapidement des préoccupations majeures dont je ne me serais sans doute pas souciée si j'avais eu le profil scolaire habituel.

Finalement, j'ai étudié au bon moment, quand je pouvais exercer mon esprit critique. Je n'aurais pas pu écrire L'amour de la carte postale ni surtout L'échappée des discours de l'œeil à 17 ans, 20 ans, 30 ans. Là, j'aurais été en extase devant Levi-Strauss, Freud, devant tous les grands écrivains. Dans ma vie, j'ai toujours souhaité que la théorie et le réel soient cohérents. Je ne trouvais pas cohérent, par exemple, qu'un professeur qui n'avait jamais mis les pieds en Europe de l'Est s'emballe pour le marxisme sans avoir fait une heure d'usine, une heure de travail dans les champs ou de travail communautaire. De quoi parlait-on? Le savoir est incomplet sans l'expérience.

V. et I. : Votre œuvre se caractérise par une très grande hétérogénéité de thèmes, de styles, mais surtout de genres littéraires: roman, poésie, pièce de théâtre, essai et journal intime. Qu'est-ce qui vous a menée à passer d'un genre à l'autre? 
M. O.-M. : Je ne le sais pas, en fait. Comme j'ai toujours écrit sous le coup d'une urgence, je suis allée là où l'urgence était, vers la nouvelle, vers le roman, vers l'essai, vers la poésie. Après coup, avec une certaine distance, je peux essayer de décanter ce qui s'est passé. Sans doute que tout cela a été un champ de travail complémentaire.

J'ai d'abord commencé par la nouvelle et le roman, forme d'écriture qui me séduit le plus parce que c'est le monde organique le plus global. Mes premiers romans posent la question de l'identité et la réponse à cette question est la tentation de la folie, de la mort, de même qu'un grand désir de création. Peut-être que mes romans les plus récents la posent encore, mais de façon beaucoup moins douloureuse.

Après Le plat de lentilles, où la protagoniste - une femme qui veut peindre - accède à une conscience qu'on pourrait appeler féminine, j'ai ressenti le besoin de passer à l'essai. Presque tout ce que j'avais reçu en fiction et en essai, de l'expression du désir au rapport au monde, était sur un mode masculin. C'était ce qui avait construit mon imaginaire toute petite, puisqu'il n'y avait pas beaucoup de livres écrits par des femmes en dehors de la comtesse de Ségur avec sa petite Sophie. J'ai donc éprouvé le désir d'aller voir pourquoi la version femme manquait et pourquoi, lorsqu'elle était là, elle fonctionnait souvent sur un mode mimétique. Le plat de lentilles avait creusé une fissure en moi et j'avais besoin de la combler. Alors, je suis allée du côté de l'essai; non pas pour raconter ce que je savais, mais pour apprendre ce que je ne savais pas. C'était d'autant plus motivant que cette question était pratiquement une matière vierge ici. Il y avait eu de courts essais publiés dans des revues, mais la question féministe avait été abordée surtout dans le langage poétique. Après L'échappée des discours de l'œeil, je suis revenue au roman, car je me sentais plus éclairée, plus libre.

V. et I. : Dans Le plat de lentilles et dans La fête du désir, vous interrogez les mystères de la vie intime, notamment le désir et l'amour, par le biais d'un style lyrique très intense. Par contre, dans L'échappée des discours de l'oeil et dans L'amour de la carte postale, vous examinez certains discours socioculturels émis par le patriarcat et l'institution littéraire, en adoptant un ton contestataire, ironique, parfois mordant. Comment expliquez-vous cette coprésence de sujets et de styles aussi variés? À la limite, on pourrait dire qu'il y a des aspects opposés dans votre œuvre.

M. O.-M. : . . p pour un esprit rationnel. Je vois plutôt cela comme complémentaire. Ces registres répondent à l'impulsion du moment, à la nature du genre littéraire traité. L'essai permet une plus grande mise à distance que le roman, tout particulièrement le roman écrit à la première personne. On gagne plus facilement dans l'essai que dans le roman, et c'est pourquoi je trouve l'essai un peu pervers. Il construit sa vérité, des idées générales, alors que le roman construit la vie, un milieu ou un moment particulier, 
une existence singulière. Je crois, malgré tout, que mon écriture n'a pas été assez mobile, qu'elle aurait pu l'être davantage. Du lyrisme, il y en a aussi dans L'échappée des discours de l'œil, et les féministes me l'ont d'ailleurs reproché. Écrire, vous savez, c'est vraiment un sauve-qui-peut. J'essaie d'écrire sans censure, en laissant venir ce qui veut se dire. J'écris rapidement et c'est seulement lors de la deuxième, troisième ou quatrième version que je commence un peu à éliminer, décanter. Je travaille sans plan même pour mes essais. Cette écriture, très pulsionnelle, va parfois selon l'humeur du moment, mais aussi selon le sujet traité.

Quand j'ai écrit L'échappée des discours de l'œil et ensuite L'amour de la carte postale, il y avait beaucoup de colère en moi, des colères, mon Dieu, refoulées depuis l'enfance, qui n'avaient jamais éclaté, parce que j'avais toujours été une enfant douce. J'ai même été une adulte douce. Maintenant, je suis très impatiente. Je suis passée de l'autre côté, je ne peux même pas dire $d u$ miroir, parce que c'était une sagesse imposée. En touchant à des discours et à des théories qui dictent les comportements, la hiérarchisation sexuelle et sociale, j'ai éveillé des colères, non contre des individus, ni contre l'homme réel, mais contre des structures sociales qui avaient tellement aplati la spontanéité et la créativité des femmes. Naturellement, pour exprimer la complexité de ces phénomènes sociaux, mon écriture a pris divers tons : la parodie, l'éclat de rire, le lyrisme, etc.

V. et I. : Je constate que, dans plusieurs de vos textes, il y a une recherche de nouvelles formes d'écriture. Par exemple, dans La maison Trestler, il y a dédoublement de la voix narrative, fragmentation du temps et de l'espace; dans La tentation de dire, on trouve une juxtaposition de différents discours, comme des fragments du journal intime de la grand-mère, des lettres d'amis et des cartes postales. Ces stratégies d'écriture, qui visent fréquemment la déconstruction des grands savoirs, intéressent vivement les critiques littéraires. Or, de ce point de vue, L'été de l'île de Grâce a étonné certains critiques à cause de son style et de son idéologie traditionnels. Pourquoi ce retour à une forme d'écriture classique?

M. O.-M.: Il y a certainement eu une régression dans l'écriture, peut-être même au niveau de l'idéologie. Dans L'été de l'île de Grâce, je pensais pouvoir reprendre les formes d'écriture expérimentées dans La fête $d u$ désir et rendues plus visibles dans La maison Trestler. J'ai même commencé la première version en essayant de le faire. Mais je me suis trouvée totalement démunie à la fois comme écrivain et comme être humain, totalement dépassée. Il y avait trop de malheur, trop d'exil et de détresse. Ce n'était plus une famille, mais des milliers et des milliers de personnes à intégrer. Alors, j'ai dû consentir à être modeste, renoncer au narcissisme, car ce n'est pas agréable de revenir à une forme d'écriture antérieure, celle que l'on ne pratique plus depuis quatre ou cinq livres. Cela a été pour moi une leçon d'humilité. C'est mon livre le plus humble et le plus généreux, celui que beaucoup de gens préfèrent, même les intellectuels, 
mais pas nécessairement les critiques. Les critiques de presse, pour la plupart, ont aimé ce livre alors que les critiques universitaires étaient en droit de me poser la question que vous soulevez. Cela a été le livre de l'impuissance, si je puis dire. Je n'ai pas pu faire les déplacements de sens, de langage, de temps et d'espace effectués dans mes autres textes.

Dans La maison Trestler, il s'agissait moins d'évoquer l'histoire avec un grand $\mathrm{H}$ que de superposer, télescoper des temps et des espaces différents: l'Europe, l'Allemagne, la France, l'Amérique du Nord. Ce qui m'intéressait, c'était de rendre la simultanéité des événements, des souvenirs qui peuvent être présents à la conscience, surtout la conscience créatrice, et d'en montrer la superposition plutôt que le déroulement. C'est pourquoi on était tantôt dans le $\mathrm{XVIII}^{\mathrm{e}}$ ou le $\mathrm{xIx}^{\mathrm{e}}$ siècle, tantôt dans le $\mathrm{xx}^{\mathrm{e}}$. L'éclatement visait à produire la simultanéité et c'était relativement facile: le monde représenté par le microcosme de la famille Trestler était plus étroit que celui de L'été de l'île de Grâce.

V. et I.: Dans L'été de l'île de Grâce, il y a un changement, par rapport à vos autres textes, de la fonction symbolique du personnage féminin. Non seulement y a-t-il perte du je narratif féminin, si fécond dans La maison Trestler, mais la femme a finalement peu d'importance dans le récit. C'est le docteur Milroy qui est le véritable héros du roman alors que son épouse, Agnès Frémont, et la servante Persévérance détiennent des rôles secondaires et traditionnels : contribuer à la santé et au bonheur du docteur. Est-ce que cela signifie que, sur le plan symbolique, il y a un gommage du féminin dans le roman et un retour au personnage féminin traditionnel?

M. O.-M.: Je ne crois pas. L'épouse du $\mathrm{D}^{\mathrm{r}}$ Milroy doit rester à Québec à cause de la loi de la quarantaine, mais elle comprend la puissance de la transgression qu'elle pratique en subvertissant, par exemple, des rituels mondains. Persévérance, c'est en fait le deuxième médecin de l'île. Elle exerce la part occulte de la médecine par ses cuisines d'herbes, ces sirops qu'elle invente, les exercices en plein air. Dans ce roman, la subversion se fait plutôt au niveau du personnage, des situations, de l'imaginaire. Il ne faut pas oublier que tout cela se déroule il y a 150 ans. Persévérance est une femme qui n'a pas de nom, elle n'a qu'un prénom assez approprié d'ailleurs. Mais puisqu'elle n'a ni profession ni scolarisation, cela n'aurait pas été simple de la laisser assumer le récit.

Dans ce roman assez traditionnel, qu'est-ce que Persévérance propose? Trois choses : l'éthique du savoir, l'éthique de la différence, la célébration de l'imaginaire. L'éthique du savoir est particulièrement visible dans la scène du microscope. Un chimiste, qui était censé avoir découvert un fluide miraculeux, descend sur l'île et initie Persévérance au microscope. Pour elle, qui s'émerveille de tout, cet instrument, qui lui fait voir des myriades de couleurs et de formes, est d'abord fabuleux. Mais 
ensuite, une frayeur la prend. Elle craint de ne plus se contenter de voir les choses telles qu'elles le sont. Surtout, elle se dit que cet instrument-là, qui peut probablement voir les consciences, donne trop de pouvoir. Elle soulève ainsi la question de l'éthique médicale et de l'éthique du savoir. Est-ce qu'on peut tout savoir? Est-ce qu'on peut exercer le pouvoir scientifique sur n'importe quoi, n'importe qui? Cela n'a l'air de rien, mais elle pose une question très grave qui préoccupe beaucoup cette fin de siècle.

Il en est de même pour la notion du respect de la différence. Persévérance voit arriver des milliers d'immigrants qui ne parlent ni français ni anglais (les Irlandais de l'époque parlaient le gaélique) et elle essaie de les comprendre. Elle a une conscience cosmique, une conception universelle du monde bien avant que n'apparaisse le cosmopolitisme. Sous des apparences très humbles, sa vie est une célébration de l'imaginaire, et cette célébration a peut être joué un rôle capital dans la vie des femmes à travers les âges.

L'idéologie traditionnelle, je trouve que Persévérance et le $\mathrm{D}^{\mathrm{r}}$ Milroy, qui n'est pas du tout à l'image du médecin traditionnel, la désamorcent conplètement.

V. et I.: Vous aimez beaucoup Persévérance...

M. O.-M.: Je l'adore. Et c'est très drôle car les journalistes l'ont vue comme étant très attirante. Le roman dit pourtant qu'elle a été dépossédée de tout, y compris de sa beauté. Des journalistes de trente ans l'ont imaginée jeune et belle.

V. et I.: En tant que personnage féminin, surtout de la façon que vous venez de la décrire, Persévérance s'inscrit dans une des grandes problématiques de votre œuvre, à savoir l'identité personnelle et socioculturelle de la femme. Quel rapport voyez-vous entre vos écrits théoriques et fictifs du point de vue de cette question?

M. O.-M. : Il y a des liens que j'ai créés inconsciemment en allant tantôt du côté de la théorie, tantôt du côté de la fiction. C'était pour moi une façon de réconcilier la théorie et la réalité concrète. Comme la traversée du réel avait précédé mes livres, j'avais constamment besoin, après ou avant d'aller dans la théorie, de faire la jonction entre ces deux pôles. Il me semblait que ni le savoir seul ni la théorie seule ne suffisaient à expliquer les phénomènes sociaux. Finalement, l'abstrait est un peu expéditif et peut être facilement concensuel: on peut tout démontrer, tout faire fonctionner sur papier. La partie difficile, c'est la réalité crue. Je croyais qu'uniquement le roman pouvait tout intégrer, pouvait illustrer à la fois le réel, le rapport à l'autre et le rapport au monde dans son ensemble; que seul le roman pouvait faire éprouver la souffrance, les contradictions. L'essai peut décrire, mais il ne fera jamais ressentir la douleur, le désir, la peur ou même la mort. 
V. et I.: Dans La tentation de dire, vous soulignez l'importance de la mémoire et des généalogies en accordant un rôle important au cahier de votre grand-mère maternelle. Dans La maison Trestler, la protagoniste entreprend des recherches fouillées sur la famille qui a habité cette demeure au début du dix-neuvième siècle. Comment cette prise de conscience du passé s'inscrit-elle, pour vous, dans ce qu'on pourrait appeler une poétique féminine?

M. O.-M. : Je pense que l'identité a beaucoup à voir avec l'image que l'on a de soi, mais l'image qu'on a de soi se nourrit d'une mémoire et d'une conscience. S'il n'y a rien eu avant, si l'enfant n'a personne qu'il peut regarder, aimer, et de qui il peut recevoir regard et amour, il ne saura jamais qui il est lui-même, il ne pourra jamais dire un je, ou un $t u$. La mémoire et la conscience de ce que l'on est, et de ce qu'on a été, contribuent à la formation de l'identité. La femme - comme groupe social - a une mémoire altérée, pleine de trous, une mémoire incertaine et une conscience parfois absente, comme chez Marguerite Duras et tant d'autres. L'histoire et le savoir ne lui ont pas restitué grand-chose sur ce plan-là, puisqu'elle était censée se limiter au biologique et au naturel. Mes essais disent qu'il y a danger de séparer trop radicalement le culturel et le naturel, le culturel tendant toujours à s'approprier le naturel et à le figer. On a besoin des deux.

L'histoire des femmes est en train de se constituer, de s'inscrire dans les savoirs et l'institution littéraire. Comme d'autres écrivaines, j'ai senti le besoin d'être à l'écoute de cette mémoire et d'en restituer une partie dans mes fictions. Mes essais cherchent plutôt à savoir comment, pourquoi et jusqu'où on avait occulté, faussé la mémoire des femmes et leurs réalisations culturelles. C'est un travail considérable qui m'a demandé beaucoup de recherches, d'autant plus que je travaillais dans le multidisciplinaire. Mais j'aurais voulu arriver à l'étape suivante: montrer aussi comment les femmes avaient été créatives. Il semble que Julia Kristeva soit en train de traiter cette question maintenant et d'illustrer le génie des femmes. J'y ai touché de façon fragmentaire, mais cela n'a pas été fait de façon systématique. Dans mes fictions, pour compenser ce manque, j'ai au moins abordé ma généalogie proche, ma mère, ma grand-mère; en un sens, c'était toucher à la généalogie de chaque femme.

Dans La tentation de dire, j'ai raconté le choc éprouvé quand j'ai trouvé une toute petite partie du journal de ma grand-mère maternelle dans une vieille valise d'un sous-sol, alors qu'elle avait écrit des pages et des pages, peut-être perdues, peut-être brûlées. La même chose est arrivée à une multitude de femmes incluant des femmes célèbres. Le journal de Virginia Woolf, on ne l'a pas eu de façon intégrale à la première publication. Le journal d'Alice James a été détruit par son frère Henry, qui a cependant conservé ses carnets à lui. Si je n'avais pas vu ma mère et ma grand-mère écrire, je ne serais probablement jamais venue à l'écriture. 
V. et I.: En dépit de l'importance que vous accordez à la question de la femme dans vos textes, vous refusez l'étiquette de féministe. Dans un de vos essais, vous avez affirmé : " $[\ldots]$ en ce qui nous concerne, nous demander s'il existe une critique féministe équivaut à nous demander s'il peut exister une littérature, une architecture, une peinture ou une musique féministe... À cette question, je réponds non ${ }^{1}$." Comment expliquez-vous cette prise de position en fonction de la thématique féminine dans votre œuvre?

M. O.-M.: Je trouve que toute étiquette est réductrice. Être féministe, nationaliste, immigrante, être la petite fille pauvre du deuxième rang que j'ai été longtemps fige l'identité. Cela réduit l'espoir, la globalité de la vie, d'une action, d'un tempérament, d'une phase considérée comme provisoire. Je préfère appliquer le qualificatif de féministe à un mouvement, à un engagement, plutôt qu'à l'art. Quand j'ai utilisé le mot parfois, c'était surtout en fonction de féministes qui réclamaient cette dénomination. Personnellement, je n'aime pas la donner et quand je le fais, c'est un raccourci. La littérature, l'art, est un substantif qui n'a pas besoin d'épithète.

Par ailleurs, caractériser l'écriture des femmes par l'étiquette féministe perpétue la ghettoïsation, encourage à faire des anthologies où l'on traite d'abord les grands auteurs et où l'on renvoie à la fin, dans un tout petit chapitre ou un petit paragraphe, à ce qu'on appelle l'écriture des femmes, l'écriture féministe. Moi, j'ai hâte que tout cela finisse.

V. et I.: Dans L'amour de la carte postale, vous démontrez, avec beaucoup d'exemples à l'appui, qu'il y a eu marginalisation de la femme dans la littérature depuis des siècles. Croyez-vous que de nos jours on peut toujours parler, dans le cadre de la littérature québécoise, de la "dissolution de la femme écrivain dans l'institution ${ }^{2}$ " ou bien pensez-vous que cette situation a changé?

M. O.-M.: La situation a changé, heureusement. Sur le plan médiatique, on marginalise de moins en moins. Mais d'un homme qui a une œuvre importante, on affirme que c'est "colossal", et de cet homme on dit la plupart du temps "quelle culture!». On entend rarement dire ça d'une femme. À valeur égale, on connaît aussi moins l'œuvre d'une femme que celle d'un homme. Pour la femme, c'est un peu comme si c'était toujours son dernier et unique livre. Il est certain que plus la société devient mixte, dans ses structures économiques, sociales et éducatives, plus il y a de femmes qui enseignent au niveau supérieur ou dirigent des revues culturelles, plus on entendra parler de la production culturelle des femmes. Il y a eu d'énormes progrès, mais les femmes sont encore trop peu

1. Madeleine Ouellette-Michalska, "La critique littéraire, ou l'écriture de la transparence", Barbara Godard (dir.), Gynocritics/La gynocritique, Toronto, ECW Press, 1987, p. 43.

2. Id., L'amour de la carte postale, Montréal, Québec/Amérique, 1987, p. 221. 
nombreuses à l'échelle supérieure de l'institution. Il se fait des choses incroyables dans certaines universités, dans certains cegeps, lieux importants de diffusion de la culture. C'est donc une question de temps, mais vous savez, il y a tellement de retard à rattraper.

V. et I. : L'histoire prend une très grande place dans votre œuvre, surtout dans La maison Trestler et dans L'été de l'île de Grâce. Vous semblez avoir une passion pour l'histoire, mais en même temps vous contestez ses discours en critiquant par exemple l'occultation de la vie quotidienne et du rôle de la femme dans les manuels. Comment voyez-vous votre rapport à l'histoire?

M. O.-M. : C'est un rapport assez passionnel, comme vous dites, fait d'attraction et de répulsion. L'histoire me répulse par sa violence, mais elle me fascine comme récit des origines, comme récit tout court. J'ébranle un peu sa crédibilité en demandant: est-ce que le récit historique ne fonctionne pas comme n'importe quel autre récit, n'obéit pas aux règles propres à tout processus narratif? Qu'est-ce que les choix historiques d'une communauté représentent comme idéologie? Cela revient à demander : comment l'idéologie se met-elle en représentation dans l'histoire? Je trouve, par ailleurs, passionnant de voir comment la mémoire corporelle tente de déjouer ce que j'appelle la mémoire d'archive, comment elle la contredit souvent, s'appliquant à la subvertir.

Mes premières expériences de lecture étaient très ritualisées. Je crois que dans mes livres, l'histoire c'est en quelque sorte le mythe ritualisé. Mon intérêt pour l'histoire est davantage relié à mon besoin d'explorer les dimensions du temps et de l'espace. Explorer le temps est fondamental pour la conscience créatrice. J'ai voulu approfondir la capacité du temps fictif de se déployer en quelques minutes ou de plonger à travers les temps de l'humanité. D'abord ceux qui nous sont proches et qui s'ouvrent ensuite à une échelle universelle et cosmique. Et puis, je crois qu'on est toujours affecté par l'histoire, un peu comme l'est Catherine Trestler dans La maison Trestler ou Persévérance dans L'été de l'île de Grâce.

V. et I. : Marilyn Randall fait remarquer dans son article pour ce dossier que les manuels d'histoire québécois parlent très peu de la terrible épidémie de typhus, en 1847 , et de la quarantaine à Grosse île. Êtes-vous d'accord avec ce commentaire et comment l'expliquez-vous?

M. O.-M.: Je ne suis pas allée vérifier, mais probablement que l'on en parle très peu. Vous en parlez beaucoup au Canada anglais?

V. et I. : Selon Randall, il y a des textes écrits en anglais consacrés à l'épidémie parce que ce phénomène fait partie de l'histoire de l'Irlande et de l'Angleterre.

M. O.-M.: Probablement que la langue anglaise a permis un transfert. Ici, le transfert ne s'est sans doute pas fait à cause de l'anglais. Mais en 
dehors des questions de langue, je pense que ce drame appartient aux franges de l'histoire. L'histoire n'est pas du tout dans le corps à corps, elle est dans le récit des gains et des pertes de pouvoir. Elle ne veut rien savoir du corps, rien savoir par conséquent de ses morts et elle n'insère pas, ou très peu, les phénomènes migratoires. Elle laisse à la fiction le soin d'assumer ses avatars. Elle laisse le roman intégrer les expériences humaines qu'elle ne prend pas en charge ou qu'elle n'a pas pris en charge assez vite.

V. et I. : Vous abordez dans plusieurs textes la question de l'intime. Dans La tentation de dire, le mot intime revient très souvent en fonction du journal. Est-ce qu'il y a pour vous un clivage ou bien une articulation entre l'intime et le social?

M. O.-M. : Difficile question. En Occident, l'articulation devient de plus en plus marquée, en partie à cause de l'influence des femmes et, peut-être surtout, à cause du désenchantement de la sphère publique, qui incite à revenir aux valeurs intimes. Si on parle de notre vie quotidienne, l'intime y est présent à chaque heure; en même temps, nous sommes des êtres sociaux et culturels. Vous donnez vos cours, vous écrivez des articles, vous faites de la recherche, mais vous restez une femme ancrée dans la vie quotidienne, dans votre souci, par exemple, des bons repas, du bienêtre de vos proches. La littérature peut représenter ces deux mondes différents, mais complémentaires. Pour ma part, je suis très heureuse quand j'arrive à les représenter dans un mouvement simultané.

V. et I. : Voyez-vous des rapports entre l'intime et un autre grand thème qui traverse votre œuvre, la question de l'origine?

M. O.-M. : Je ne l'ai pas vu en écrivant, mais après coup, c'est évident. L'origine m'a fascinée dans le double rôle qu'on lui a fait jouer aux plans social et personnel. Au plan social, l'origine c'est ce qui a assuré la légitimité du pouvoir dans les sociétés traditionnelles. C'était donc important de contrôler la femme, qui garantissait le pouvoir biologiquement, par des lois, des théories dictant sa nature, son comportement, sa pensée. La philosophie, l'histoire, la psychanalyse, la littérature et la langue ont toutes entériné ce processus.

Cette nécessité obligeait en un certain sens à laisser la femme du côté de la nature, tandis que l'homme s'ouvrait au culturel. Situer l'intime et le social, c'est poser les rapports nature-culture et établir des lieux de démarcation très nets. L'interdiction de l'inceste, les rapports de parenté ont structuré non seulement les sociétés dites primitives, mais également les nôtres en matière de dot, d'échange, de contrôle corporel, de ceinture de chasteté, par exemple. On lève des hauts cris contre l'excision, et il est vrai que c'est odieux, mais la ceinture de chasteté au Moyen Âge occidental n'était sans doute pas très confortable... L'absence des femmes de la culture est donc en grande partie due à l'organisation de sociétés qui, 
depuis des siècles, séparent nature et culture. C'est en partie ce que j'ai voulu démontrer dans L'échappée des discours de l'œeil.

V. et I. : Vous parlez aussi, dans vos textes de fiction, du lien entre l'origine et le désir.

M. O.-M.: Oui, j'avais depuis longtemps la curiosité de remonter à l'origine du désir dans la vie psychique. Non pas pour toucher l'origine en tant que telle, car une femme comprend facilement que la vie puisse venir d'un corps de femme. Mais la question de l'origine est en grande partie la question des possibles. Est-ce qu'il n'aurait pas pu y avoir d'autres histoires que celles qui se sont déroulées individuellement et collectivement? Est-ce que chacun de nous a actualisé ou actualise tous ses possibles? Il me semble que ce qui pourrait être fait à partir d'un corps, d'une intelligence, d'une pensée, d'un groupe humain est infini. En fin de compte, on ne réalise qu'une infime part de soi. D'où le sens de La maison Trestler ou plutôt du $\mathcal{8}^{e}$ jour: le $\boldsymbol{g}^{e}$ jour non encore inventé. Voilà ce que pour moi l'origine veut dire: effacer les traces reçues, revenir à une sorte de matrice vierge, repartir à zéro et permettre l'accomplissement des possibles demeurés latents. La fascination de l'origine est le sujet principal de La fête $d u$ désir, qui exprime le désir du meurtre symbolique des parents en nous-mêmes; le désir de revenir au début, de se remettre au monde. L'utopie est belle et impossible ...

V. et I. : Quel est l'impact, si impact il y a, des comptes rendus et des articles sur votre œuvre, sur votre écriture?

M. O.-M. : C'est surtout un impact émotif. Un geste d'accompagnement si la critique est bonne. Écrire, c'est jeter une lettre à la poste, c'est un geste amoureux que l'on veut fusionnel avec le monde, avec ses personnages, avec le thème développé. Si on reçoit une première lecture qui est aussi une lettre d'amour, on est ravi. La correspondance a été complète même si on n'écrit pas pour les autres. C'est absurde de dire cela puisque, si l'on n'était pas publié, on n'écrirait sans doute pas longtemps. Mais à l'instant où l'on écrit, on ne pense pas aux autres, on pense égoïstement: "Sauvequi-peut, laissons dégorger ce qui veut se dire." Donc c'est toujours un plaisir quand une critique est bienveillante, accueillante, intelligente, créative. Une critique bien faite, c'est un geste créateur, un autre lieu de création qui me fait énormément plaisir.

Vous savez, on est vulnérable à la sortie d'un livre. On a ouvert parfois des lieux difficiles en soi; en plus, il y a la fatigue physique si on fait une campagne de presse en même temps. On peut avoir beaucoup de peine, on peut être très heureux ou profondément blessé, déçu parce qu'on est dans un état de fragilité. On aurait besoin de beaucoup de silence, d'une grande solitude parce que écrire est un acte rigoureusement privé; un acte de réflexion et de méditation. Et puis tout à coup, on passe sur la place publique et on ne cesse de parler. Pour moi, ce passage est très difficile à 
faire. Une bonne critique aide à faire ce passage, mais une critique inintelligente, méchante, obtuse, révèle moins l'auteur ou le contenu d'un livre que l'auteur du compte rendu. Plus tardivement, la critique institutionnelle ouvre d'autres portes, non destinées au même public, et elle continue le travail de création. Elle fait connaître des thèmes, donne un autre sens à l'œuvre, établit des points de rencontre entre des auteurs, des tendances, des époques. Finalement, l'œuvre elle-même, c'est le temps seul qui déterminera son oubli ou sa prolongation. Mais les travaux critiques sont des voix d'accompagnement dans la création et la diffusion.

V. et I. : Pouvez-vous me parler de votre prochain roman et de vos projets d'écriture?

M. O.-M. : J'ai des projets pour deux livres, mais je vous dis tout de suite que, depuis L'été de l'île de Grâce, je ne suis plus dans l'écriture de la passion, de l'urgence, là où j'ai toujours été. Je crois être arrivée à une autre étape. Ce ne serait pas dramatique si je cessais d'écrire. En tous les cas, ce n'est plus nécessaire à ma survie, alors que certains livres m'ont aidée à survivre.

V. et I. : Mais vous préparez quand même un roman. J'ai bien vu le titre d'un roman à paraître dans notre bibliographie, La passagère. N'avez-vous pas envie d'en parler?

M. O.-M.: Ce roman, je l'ai écrit dans la sérénité et non dans la passion. Cela m'a dérangée, car la motivation fondamentale n'était plus là. C'est comme si j'avais maintenant moins besoin du symbolique pour résoudre les contradictions, les petits et les grands drames de la vie. Et puis il y a eu la naissance de mes deux petits-enfants, qui sont la beauté et la vie mêmes. C'est si extraordinaire ce renouvellement de la vie que les mots deviennent moins importants.

V. et I. : Vous allez quand même le publier, ce roman?

M. O.-M. : Vous tenez vraiment à ce que je vous en parle... La passagère paraîtra bientôt. J'ai commencé ce roman paresseusement à partir de brèves notes de voyage, car je n'avais plus le courage d'inventer un roman de $\mathrm{A}$ à $\mathrm{Z}$. Ce livre traverse divers lieux de l'espace. Alors vous voyez venir l'interrogation sur le temps, n'est-ce pas? Il s'agit d'une femme de 40 ans qui quitte l'homme qu'elle aimait, et qui l'aimait. Elle part tout simplement parce qu'elle a besoin de partir, de rester libre, de s'appartenir, de "danser sa vie", comme le disait La danse de l'amante. Mais le corps a la mémoire longue, l'amour que l'on quitte ne nous quitte pas aussi facilement.

Tous les grands thèmes récurrents de mon écriture sont présents dans ce roman : la mémoire, la fragilité du désir, la douleur du monde et parfois sa bêtise. Cette femme n'est pas malheureuse, mais elle sent la douleur du monde là où elle passe à cause de certains événements très précis et du relativisme des cultures. Les questions fondamentales qui me hantent depuis toujours reviennent dans ce livre, mais avec plus de sérénité. 